\title{
¿Chateamos? Motivaciones del uso de las apps de citas en el colectivo LGB
}

\section{Do we chat? Motivation for using dating apps in LGB community}

Fecha de recepción: 24-07-2020

Fecha de aceptación: 23-11-2020

\author{
Cristina Giménez-García \\ Salusex. Dpto. Psicología Básica, Clínica y Psicobiología. \\ Universitat Jaume I de Castelló. España. \\ Naiara Martínez-Gómez. \\ Salusex. Dpto. Psicología Básica, Clínica y Psicobiología \\ Universitat Jaume I de Castelló. España. \\ Marcel Elipe-Miravet. \\ Salusex. Dpto. Psicología Básica, Clínica y Psicobiología. \\ Universitat Jaume I de Castelló.España.
}

\section{resumen/ahstract:}

Las aplicaciones de citas suponen un nuevo entorno para experimentar relaciones afectivo-sexuales, en particular entre personas lesbianas, gais y bisexuales (LGB). Por ello, se analiza el uso las aplicaciones de citas en población LGB, en comparación con población heterosexual, evaluando también las diferencias de género en el uso y la relación con otras variables como el valor otorgado a la sexualidad y sensualidad, la autoestima y la regulación emocional. En total, participaron 185 personas (65.4\% mujeres y 34.6\% hombres) con edad promedio de 29.15 años (DT = 6.91), identificándose un $44.3 \%$ como heterosexuales y un $55.7 \%$ como parte del colectivo LGB. En general, la población LGB usa en mayor medida las aplicaciones de citas y de manera más frecuente que la heterosexual. Dentro del colectivo, se observa que los hombres informan un mayor uso que las mujeres y, entre las personas usuarias, se observaría una mayor autoestima y uso del sexo para evitar el malestar emocional. Dadas las diferencias encontradas, además de la existencia de indicadores psicológicos asociados al riesgo, parece necesario incluir este escenario de interacción sexual en las estrategias de intervención preventivas y de atención clínica, desde un enfoque inclusivo y con perspectiva de género.

Dating apps provide a new context to experience affective-sexual relationships, particularly among the lesbian, gay and bisexual (LGB) community. Therefore, the use of dating applications is analyzed among LGB people, compared to heterosexual people, also evaluating the gender differences in the use and variables such as the value given to sexuality and sensuality, self-esteem, and emotional regulation. In total, 185 people participated $165.4 \%$ women and $34.6 \%$ men) with an average age of 29.15 years (SD= 6.91$)$, self-identifying $44.3 \%$ as heterosexual and $55.7 \%$ as part of the LGB community. In general, LGB participants use dating apps more and more frequently than heterosexual participants. Within the group, men report higher use than women and, the app users show higher self-esteem and the use of sex to avoid emotional distress. Given these differences, as well as the existence of psychological indicators related to risk, it seems necessary to include this context of sexual interaction in preventive strategies and clinical interventions, from an inclusive approach and gender perspective.

\section{palabras clave/keywords:}

LGB; apps; motivación de uso; regulación emocional; autoestima.

LGB; apps; motivation to use; emotional self-regulation; self-esteem. 


\section{Introducción}

En las últimas décadas, el avance de las tecnologías de la información y la comunicación (TIC) ha convertido a las redes sociales en un nuevo espacio de socialización (Ibarra y Pérez, 2013). Este fenómeno, también se ha extendido al ámbito de la sexualidad, modulando la forma en la que se desarrollan las relaciones afectivo-sexuales entre las personas (Ansari, 2015). En concreto, el uso de las aplicaciones de citas (por ejemplo, Grindr, Tinder o Wapa) está aumentando en los últimos años. Según algunos estudios, aplicaciones como Tinder tendrían 60 millones de inscripciones a nivel mundial reportando, en la actualidad, 26 millones de "match" al día (Latamclick, 2018). En concreto, en países como Estados Unidos, se ha observado un incremento notable en su uso durante los últimos cinco años, con independencia de la situación de pareja (Vogels, 2020). Tal y como recoge Chan (2017), para algunas personas, las aplicaciones añadirían un valor diferencial respecto a otros dispositivos, al posibilitar una mayor inmediatez en sus encuentros sexuales, dada la cercanía geográfica y temporal en la que se encuentran las personas con las que contactan. Al mismo tiempo, algunas personas las percibirían como más auténticas, dado que se necesita un registro para su uso, además de más sugerentes, al resultar más visuales que otras opciones como las webs de citas.

Asimismo, aquellas personas que muestran una mayor búsqueda de sensaciones sexuales, actitudes más abiertas hacia la sexualidad y perciben el uso de las aplicaciones de citas como algo normalizado y legítimo a nivel social, las usarían en mayor medida (Chan, 2017; Sevi, Aral y Eskenazi, 2018). Al mismo tiempo, entre las personas usuarias, se han encontrado actitudes más abiertas frente a la exposición al riesgo y una mayor realización de las propias conductas de riesgo (Tomaszewska y Schuster, 2020), así como el empleo de las aplicaciones de citas como un regulador emocional que amortiguaría el impacto de algunas vivencias desagradables (Martínez-Rodrigo, Segura-García y Sánchez-Martín, 2011).

Estas variables, podrían relacionarse con algunas de las consecuencias de riesgo que conllevaría el uso de las apps. En concreto, el aumento de los encuentros sexuales esporádicos con personas conocidas en línea ha facilitado un incremento de las Infecciones de Transmisión Sexual (ITS) (Bull y McFlare, 2000; Klein y Tilley, 2012). En otros casos, en los que no ha habido interacción física, los escenarios online han dificultado el desarrollo de habilidades relacionales y el establecimiento de vínculos afectivos entre personas (Weinstein, Zolek, Babkin, Cohen y Lejoyeux, 2015). A su vez, el anonimato atribuido al espacio online podría incrementar el discurso discriminatorio y violento contra algunos colectivos (Rothenburg y Stroppa, 2015) o entornos de pareja (Sabella, Patchin y Hinduja, 2013); así como promover la normalización discursiva que jerarquiza los sentidos de masculinidad y feminidad y la expresión de género (Grohmann, 2016).

Por otro lado, el uso de los entornos online también se ha relacionado con una mejora del conocimiento sexual y de la satisfacción en las relaciones de pareja, así como el cumplimiento de los deseos sexuales (Daneback, Sevcikova, Månsson y Ross, 2013). De manera específica, en colectivos como el de lesbianas, gais y bisexuales (LGB), las relaciones afectivo-sexuales a través de las apps han mostrado una mejora del bienestar afectivo-sexual, a 
través de aspectos como (Taylor, Huston y Alicea, 2017; Timmermans y De Caluwé, 2017): proporcionar un espacio más seguro para desarrollar las habilidades sociales y sexuales, facilitar una mayor privacidad en el establecimiento de contactos, favorecer la participación en un clima de mayor aceptación social, facilitar un mayor sentimiento de pertenencia y también posibilitar un mayor control en la toma de decisión sobre el ritmo de apertura y desarrollo psicosexual. Dado el contexto de exclusión y estigma que todavía permanece hacia a las minorías sexuales, las personas LGB podrían buscar en las apps, un entorno en el que desarrollar su experiencia afectivo-sexual, sin temor a sufrir el rechazo social debido a su orientación sexual (Ashford, Clifton y Kaneda, 2006). De esta forma, los espacios online y en particular las aplicaciones de citas supondrían un lugar más seguro para explorar, en las fases iniciales del proceso identitario, el cuestionamiento de forma anónima y marcar los propios ritmos del proceso de autorrevelación, facilitando una mayor autoaceptación de la orientación sexual (Taylor et al., 2017).

En este sentido, las aplicaciones de citas presentarían un nuevo paradigma para la comunidad LGB, lo que podría incrementar su uso respecto a otras poblaciones. Según el estudio de Johnson, Vilceanu y Pontes (2017), en Estados Unidos, un 29.8\% de población LGB usaría aplicaciones de citas, frente a un $10.5 \%$ de población heterosexual. Este fenómeno, resultaría una extensión de lo observado en otros entornos. Tal y como ilustra el estudio de Smith (2016), el 15\% de las parejas heterosexuales se conocerían a través de internet, mientras que lo haría el $70 \%$ de las parejas homosexuales.

A su vez, dentro de este colectivo, algunos estudios han mostrado ciertas diferencias de género. En concreto, en España, los hombres realizarían un mayor uso que las mujeres y también informarían de diferencias en aspectos específicos como haber mentido en alguna ocasión o el tipo de imágenes en el que se fijan (Martínez-Gómez, Cervigón-Carrasco, Nicola-Piris y Giménez-García, 2020). Otras diferencias se relacionarían con las motivaciones de uso, siendo los hombres los que informarían una mayor motivación de uso relacionada con los encuentros casuales (Sumter, Vandenbosch y Ligtenberg, 2017). Asimismo, el estudio de Hamilton, Franklin, Condesa y Cuauhtémoc (2018) también mostró diferencias cualitativas, entre hombres y mujeres, en el uso de las aplicaciones y la creación de perfiles. En estos últimos, las mujeres destacarían los rasgos faciales, así como la inteligencia y la lealtad, frente a los hombres que destacarían los rasgos corporales, el liderazgo y el humor. Además, mientras que los hombres informaban fijarse en las fotografías, las mujeres se centraban en las descripciones.

Todos estos estudios, pondrían de manifiesto la importancia de la orientación sexual y el género en la experiencia de las apps de citas, si bien se evidencia que se ha desatendido aspectos tan relevantes como la normalización de su uso al comentarlo con otras personas, la posibilidad de realizar conductas offline tras el uso de las apps o la influencia de factores como la regulación emocional. Por este motivo y dada la importancia que el uso de las apps de citas podría tener para el bienestar sexual de la población e incluso para la exposición ante riesgos sexuales, parece fundamental explorar con mayor detalle, tanto en hombres como en mujeres LGB, el uso de las apps de citas. 
Por lo tanto, el presente estudio busca analizar el patrón de uso y motivaciones de las apps de citas en población LGB, en comparación con la población heterosexual, así como valorar posibles diferencias en función del género. Además, en la población LGB, también se estudia la diferencia que existe entre personas usuarias y no usuarias de apps en algunas variables psicosexuales tan relevantes como la importancia otorgada a la sexualidad y la sensualidad, la autoestima y la regulación emocional.

\section{Método}

\section{Participantes}

En este estudio han participado 185 personas, $65.4 \%$ mujeres y $34.6 \%$ hombres. Según la edad, tenían una media de 29.15 años $(\mathrm{DT}=6.91)$, siendo el rango de edad entre 20 y 45 años. En el momento del estudio, el 58.4\% tenía pareja estable y un $35.7 \%$ no la tenía, mientras el 5.9\% refirió no haberla tenido nunca. Por lo que respecta al tipo de relación, un $75.1 \%$ refería tenerlas monógamas, un $14.6 \%$ abiertas (relaciones donde existen prácticas sexuales fuera de la pareja estable, de forma consensuada), un 3.8\% poliamorosas (personas que establecen más de una relación afectivo sexual de forma consensuada) y un $6.5 \%$ informó que no se podía incluir entre las anteriores. Finalmente, en cuanto a la orientación sexual, un $44.3 \%$ se consideraba heterosexual y un 55.7\% parte del colectivo LGB. Dentro de este colectivo, el $35 \%$ se identificaban como homosexuales, un $21 \%$ como lesbianas y un $44 \%$ como bisexuales.

\section{Instrumentos}

Para llevar a cabo el estudio, se incluyeron varios instrumentos que posibilitaron evaluar tanto el uso de las aplicaciones, como las variables psicosexuales incluidas. A continuación, se detalla cada uno de ellos:

Cuestionario sobre uso de aplicaciones móviles. Este instrumento busca explorar la topografía del uso de aplicaciones, así como las motivaciones y factores que facilitan su uso y las consecuencias percibidas. En concreto, se exploran variables como: la frecuencia de uso, número de aplicaciones, la forma de acceso, imágenes de perfil empleadas, sinceridad y finalidad de su uso, si ha conocido a otras personas a través de las aplicaciones y si ha mantenido relaciones sexuales con ellas. En total, consta de 23 ítems, con preguntas dicotómicas y de escala Likert. Este instrumento, también incluye una sección de datos sociodemográficos que, a través de 11 ítems, recoge información acerca de la edad, identidad sexual, orientación sexual, ocupación y situación sentimental. Al mismo tiempo, se incluye un apartado relativo a la experiencia sexual en el contexto offline a través de 19 ítems. En este apartado, se pregunta a través de dos escalas Likert, de 1 (nada importante) a 4 (muy importante), el valor otorgado a la sensualidad en las relaciones sexuales y la importancia otorgada a la sexualidad en las relaciones de pareja.

Escala de autoestima sexual del Cuestionario Multidimensional sobre Sexualidad (Snell, Fisher, y Walters, 1993). Este instrumento mide de forma multidimensional diferentes as- 
pectos del funcionamiento sexual. Para el presente estudio se ha empleado la subescala de 5 ítems que mide la autoestima sexual $(\alpha=0.81)$. El formato de respuesta es de 5 puntos, entre 1 (En absoluto característico de mí) y 5 (Muy característico de mí).

Inventario de Hipersexualidad de Reid, Garos y Carpenter (2011), en su versión validada en castellano (Ballester-Arnal, Castro-Calvo, Gil-Juliá, Giménez-García y Gil-Llario, 2019). Este instrumento contiene 19 ítems para valorar la hipersexualidad, con un formato de respuesta tipo Likert entre 1 (Nunca) y 5 (Muchas veces). En concreto, para este estudio, se han empleado los 7 ítems relativos al factor "afrontamiento" $(\alpha=0.93)$ que valora el uso del sexo como regulador de emociones desagradables y los 7 ítems incluidos en el factor "Control" $(\alpha=0.91)$, que explora la presencia de déficits a la hora de controlar los pensamientos, impulsos o conductas sexuales.

\section{Procedimiento}

El estudio se difundió a través de redes sociales dirigidas a grupos generales de población, así como a colectivos LGB. En el mensaje se daba información relativa al estudio, además de un enlace a través del que podían acceder a la plataforma de investigación. Una vez accedían a la plataforma, aparecía una ventana en la que se recordaba la información del estudio, el carácter voluntario, anónimo y confidencial de su participación, así como la necesidad de dar el consentimiento informado. Tras dar dicho consentimiento, las personas podían acceder a los instrumentos de evaluación. El estudio contó con la aprobación de la Comisión Deontológica de la Universitat Jaume I.

\section{Análisis estadísticos}

Con el fin de caracterizar la muestra, se han realizado estadísticos descriptivos. Además, para conocer las diferencias entre la población heterosexual y LGB, así como entre las personas LGB que usan y no usan apps, se emplearon pruebas Chi cuadrado y V de Cramer para las variables nominales y pruebas t de Student, para las variables continuas. Los análisis estadísticos se realizaron con el programa estadístico SPSS 26.

\section{Resultados}

Comparación de uso de aplicaciones de citas entre población LGB y población heterosexual.

En primer lugar, se hizo una comparación entre la población LGB y la población heterosexual en cuanto al uso de las apps de citas. En concreto, un $63.1 \%$ de la población LGB informó utilizarlas frente al $23.2 \%$ de la población heterosexual, mostrando estas diferencias significación estadística $\left(\chi^{2}=29.37 ; \mathrm{p}<0.001 ; \mathrm{V}=0.398\right)$. Respecto a la frecuencia de uso en los últimos 3 meses, se observa que la población LGB informa una mayor frecuencia diaria y de más de 3 veces a la semana, mientras la población heterosexual lo hace en mayor medida entre 1 y 3 veces a la semana y menos de 4 veces al mes (ver Figura 1). Estas diferencias resultan significativas a nivel estadístico $\left(\chi^{2}=12.18 ; \mathrm{p}=0.016 ; \mathrm{V}=0.403\right)$. 


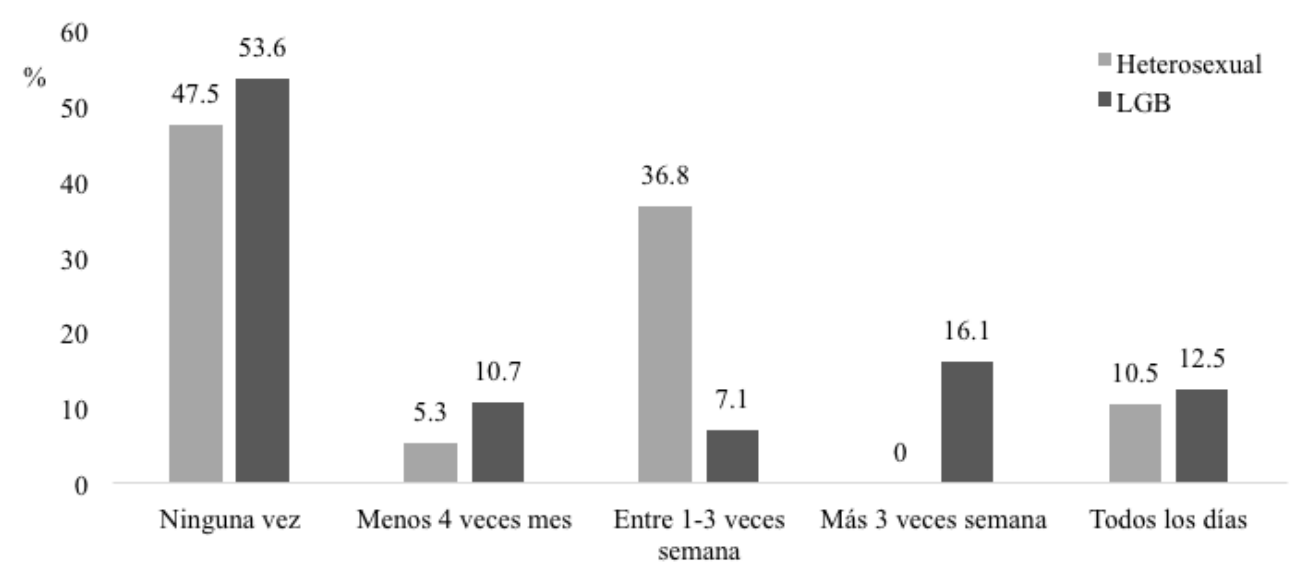

Figura 1. Distribución de frecuencias de uso de las apps según orientación sexual en los últimos tres meses

En cuanto a la posibilidad de comentar con otra persona el uso que realizan de las aplicaciones, no se observa significación a nivel estadístico $\left(\chi^{2}=3.78 ; \mathrm{p}=0.150 ; \mathrm{V}=0.212\right)$, si bien el $94.7 \%$ de población heterosexual lo compartiría con personas cercanas, frente al 75.4\% de la población LGB. A su vez, un $13.8 \%$ de esta última lo comentaría con cualquier persona, fuera o no conocida, mientras que ninguna persona heterosexual lo haría. El 5.3\% de población heterosexual y el $10.8 \%$ de las personas LGB, no lo comentarían con nadie.

Respecto a la finalidad de uso de estas aplicaciones (ver Figura 2), la población LGB muestra porcentajes superiores en casi todas las variables, destacando esta diferencia en el propósito de hacer amistades $\left(\chi^{2}=2.74 ; \mathrm{p}=0.097 ; \mathrm{V}=0.183\right)$, la curiosidad $\left(\chi^{2}=3.13 ; \mathrm{p}=\right.$ $0.077 ; \mathrm{V}=0.196)$ y entretenimiento $\left(\chi^{2}=1.74 ; \mathrm{p}=0.187 ; \mathrm{V}=0.147\right)$, aunque no exista significación a nivel estadístico. Algo similar ocurre respecto a encontrar pareja $\left(\chi^{2}=0.193\right.$; $\mathrm{p}=0.661 ; \mathrm{V}=0.048)$ y tener relaciones sexuales $\left(\chi^{2}=0.321 ; \mathrm{p}=0.571 ; \mathrm{V}=0.062\right)$, se encuentran puntuaciones similares. Por el contrario, más población heterosexual informa la intención de disminuir el estrés mediante el uso de las aplicaciones $\left(\chi^{2}=0.44 ; \mathrm{p}=0.507 ; \mathrm{V}\right.$ $=0.073)$ y la excitación sexual $\left(\chi^{2}=1.14 ; \mathrm{p}=0.285 ; \mathrm{V}=0.119\right)$, sin existir tampoco diferencias significativas a nivel estadístico.

Para finalizar los análisis de comparación en función de la orientación sexual, se evaluó si al usar las aplicaciones se había llegado a conocer físicamente a una persona y a tener relaciones sexuales con ella. Tanto las personas heterosexuales como las LGB afirman haber conocido físicamente a las personas con las que han conectado virtualmente, en concreto un $84.2 \%$ y un $89.2 \%$, respectivamente, sin existir diferencias estadísticas significativas $\left(\chi^{2}=\right.$ $0.35 ; \mathrm{p}=0.552 ; \mathrm{V}=0.065)$. Por su parte, el $63.2 \%$ de las personas heterosexuales afirman que en esos encuentros físicos practicaron conductas sexuales, mientras que lo afirma un $72.3 \%$ de la población LGB, aunque dicha diferencia tampoco resulta significativa a nivel estadístico $\left(\chi^{2}=0.58 ; \mathrm{p}=0.443 ; \mathrm{V}=0.084\right)$. 


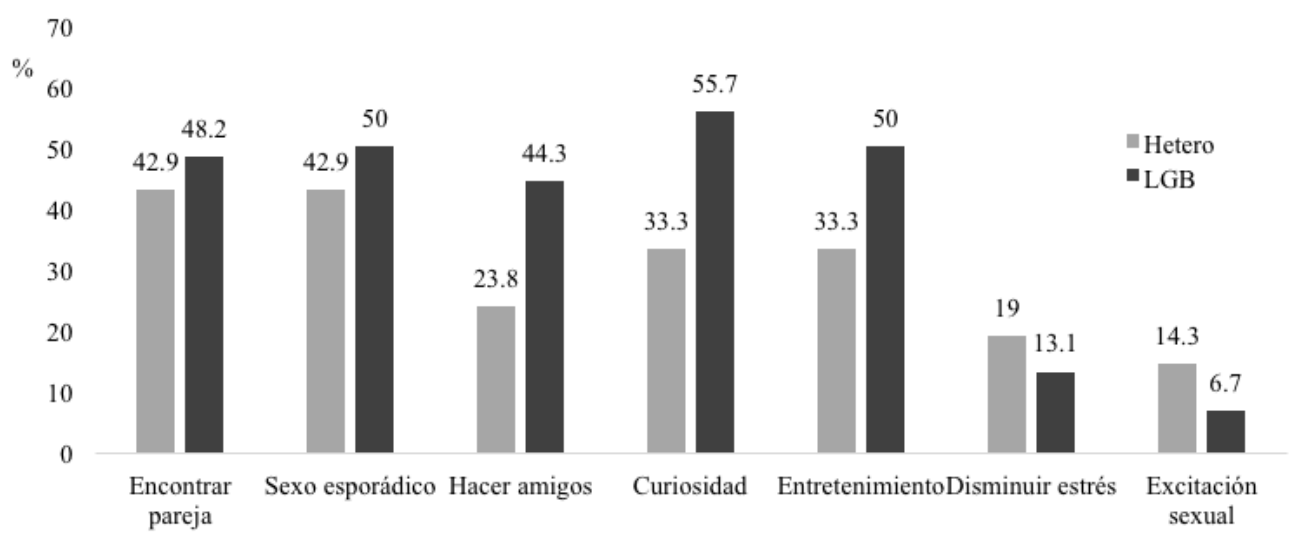

Figura 2. Distribución de frecuencias en finalidad de uso de las apps según orientación sexual

\section{Comparación de uso de apps entre hombres y mujeres $L G B$}

En cuanto al uso de aplicaciones de citas entre hombres y mujeres dentro del colectivo LGB, se observa que más hombres (71.4\% de ellos) que mujeres (58.8\% de ellas) las utilizan, no encontrándose diferencias significativas $\left(\chi^{2}=1.57 ; \mathrm{p}=0.209 ; \mathrm{V}=0.124\right)$.

En lo que a finalidad de uso se refiere (ver Figura 3), se observa que la única diferencia estadística significativa reside en que los hombres encuentran una mayor excitación sexual en el uso de las aplicaciones que las mujeres $\left(\chi^{2}=7.40 ; \mathrm{p}=0.007 ; \mathrm{V}=0.351\right)$. De hecho, ninguna de ellas la informa como finalidad para emplearlas. También queda patente que un mayor porcentaje de hombres utilizan estas aplicaciones con la intención de tener relaciones sexuales esporádicas $\left(\chi^{2}=3.38 ; \mathrm{p}=0.066 ; \mathrm{V}=0.254\right)$, hacer amigos $\left(\chi^{2}=2.24 ; \mathrm{p}=0.134 ; \mathrm{V}=\right.$ $0.192)$, encontrar pareja $\left(\chi^{2}=0.21 ; \mathrm{p}=0.647 ; \mathrm{V}=0.058\right)$ y disminuir el estrés $\left(\chi^{2}=0.59 ; \mathrm{p}=\right.$ $0.441 ; \mathrm{V}=0.099)$, aunque estas diferencias no lleguen a ser significativas a nivel estadístico. El único propósito en el cual las mujeres puntúan más alto que los hombres es el relativo al entretenimiento $\left(\chi^{2}=1.76 ; p=0.184 ; \mathrm{V}=0.171\right)$, no siendo tampoco significativas estas diferencias a nivel estadístico.

Por último, se quiso ver si existían diferencias entre hombres y mujeres en el hecho de haber conocido físicamente a alguien mediante estas aplicaciones y haber mantenido relaciones sexuales con esa persona. En cuanto a conocer físicamente a alguien, el $88 \%$ de los hombres y el $90 \%$ de las mujeres afirman haber quedado con alguna persona conocida a través de estas aplicaciones, no existiendo diferencias significativas a nivel estadístico $\left(\chi^{2}=0.64 ; \mathrm{p}\right.$ $=0.800 ; \mathrm{V}=0.031)$. Por lo que respecta a mantener relaciones sexuales con las personas que han conocido, un $76 \%$ de hombres y un $70 \%$ de mujeres afirman haber practicado sexo con ellas, sin existir diferencias estadísticas entre ambos grupos $\left(\chi^{2}=0.27 ; \mathrm{p}=0.599 ; \mathrm{V}=\right.$ $0.065)$. 


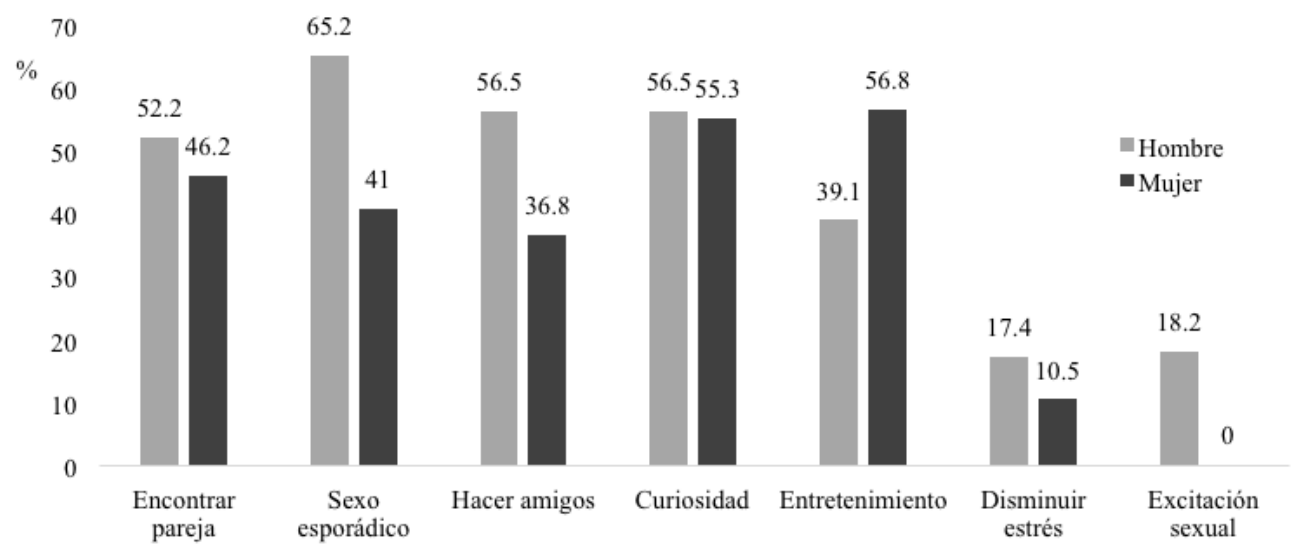

Figura 3. Distribución de frecuencias en finalidad de uso de las apps entre hombres y mujeres LGB

Diferencias en el perfil psicosexual entre personas LGB que usan y que no usan apps

En primer lugar, se quiso evaluar si existían diferencias de autoestima entre población LGB que usa apps respecto a la misma población que no las usa (ver Tabla 1). Las pruebas realizadas revelan que más personas que utilizan este tipo de aplicaciones se perciben como una pareja sexual bastante buena, respecto a quienes no usan estas aplicaciones. De igual forma, más personas usuarias de apps consideran que son mejores en el sexo que otras personas y que son unas parejas sexuales muy favorables frente a las personas que no suelen utilizarlas. Estas diferencias resultan significativas a nivel estadístico.

Tabla 1.- Análisis diferencial de la autoestima entre personas LGB que usan y no usan apps

\begin{tabular}{lcccccc}
\hline & $\begin{array}{c}\text { Uso } \\
\text { apps }\end{array}$ & $\begin{array}{c}\text { Nada } \\
\mathbf{( \% )}\end{array}$ & $\begin{array}{c}\text { Ligera- } \\
\text { mente } \\
(\mathbf{( \% )}\end{array}$ & $\begin{array}{c}\text { Modera- } \\
\text { damente } \\
\mathbf{( \% )}\end{array}$ & $\begin{array}{c}\text { Mucho } \\
\mathbf{( \% )}\end{array}$ & $\begin{array}{c}\mathbf{C h i}^{2} \\
\text { (p) }\end{array}$ \\
\hline Tengo confianza en mí & Sí & 2 & 11.8 & 33.3 & 52.9 & 5.24 \\
como compañero sexual & No & 5.9 & 29.4 & 11.8 & 52.9 & $(0.155)$ \\
Soy una pareja sexual & Sí & 3.9 & 11.8 & 41.2 & 43.1 & 11.22 \\
bastante buena & No & 5.9 & 41.2 & 47.1 & 5.9 & $(0.11)$ \\
Soy mejor en el sexo que & Sí & 21.6 & 23.5 & 35.3 & 19.6 & 10.27 \\
otras personas & No & 35.3 & 52.9 & 11.8 & 0 & $(.016)$ \\
Creo que soy una pareja & Sí & 7.8 & 11.8 & 45.1 & 35.3 & 10.12 \\
sexual muy favorable & No & 17.6 & 41.2 & 29.4 & 11.8 & $(.018)$ \\
Me siento con confianza & Sí & 9.8 & 19.6 & 31.4 & 39.2 & 7.38 \\
en los encuentros sexuales & No & 35.3 & 11.8 & 35.3 & 17.6 & $(.061)$ \\
\hline
\end{tabular}


En cuanto al papel que juega el sexo en las relaciones de pareja y el rol de la sensualidad en las relaciones sexuales, las personas usuarias de apps de citas consideran en mayor medida el sexo como algo muy importante y bastante importante dentro de la pareja, en comparación con quienes no las usan (ver Tabla 2). Estas diferencias resultan significativas a nivel estadístico. En lo que se refiere a la importancia otorgada a la sensualidad en las relaciones sexuales, no se han encontrado diferencias significativas, pues tanto usuarios como no usuarios de apps consideran este aspecto como algo muy importante y bastante importante en la sexualidad.

Tabla 2.- Análisis diferencial de la importancia del sexo y la sensualidad entre personas LGB que usan y no usan apps

\begin{tabular}{lcccccc}
\hline & $\begin{array}{c}\text { Uso } \\
\text { apps }\end{array}$ & $\begin{array}{c}\text { Nada } \\
(\mathbf{\%})\end{array}$ & $\begin{array}{c}\text { Algo } \\
\mathbf{( \% )}\end{array}$ & $\begin{array}{c}\text { Bastante } \\
\mathbf{( \% )}\end{array}$ & $\begin{array}{c}\text { Mucho } \\
\mathbf{( \% )}\end{array}$ & Chi' $^{\mathbf{2}} \mathbf{( p )}$ \\
\hline \multirow{2}{*}{ Importancia del sexo } & 2 & 3.8 & 47.1 & 47.1 & 15.82 \\
& No & 0 & 41.2 & 35.3 & 23.5 & $(0.001)$ \\
$\begin{array}{l}\text { Importancia de la } \\
\text { sensualidad }\end{array}$ & Sí & 0 & 2 & 35.3 & 62.7 & 5.50 \\
& No & 0 & 11.8 & 52.9 & 35.3 & $(.064)$ \\
\hline
\end{tabular}

A pesar de no existir diferencias significativas en el uso del sexo como regulador emocional entre personas usuarias y no usuarias de apps (ver Tabla 3), los resultados muestran que las primeras podrían usar algo más el sexo como estrategia de evitación frente a sentimientos desagradables como la ira, la frustración o la tristeza.

Al mismo tiempo, aun sin diferencias significativas a nivel estadístico, también informarían algo más de una disminución de sentimientos de soledad mediante el uso de la sexualidad.

Tabla 3.- Análisis diferencial sobre el uso del sexo como regulador emocional en personas LGB que usan y no usan apps

\section{Uso de apps}

\section{Regulación emocional}

Sí

M(DT)

Uso el sexo para olvidarme de las preocupaciones cotidianas.
$2.31(1.16) \quad 2.24(1.15) \quad-0.24(0.809)$ $\mathbf{t}(\mathbf{p})$ M(DT)

Aunque me he prometido no repetir cierta conducta sexual, me encuentro volviéndola a hacer una y otra vez.
$1.88(0.95)$
2.18(1.07)
$1.07(0.289)$ 
Hacer cualquier cosa relacionada con el sexo me ayuda a sentirme menos solo/a.

Participo en actividades sexuales sabiendo que más tarde me arrepentiré.

Recurro al sexo cuando experimento sentimientos desagradables (por ejemplo, frustración, tristeza, ira).

Fracaso en mis intentos por cambiar mi conducta sexual.

Cuando me siento inquieto, recurro al sexo para calmarme.

Practico actividades sexuales que están en contra de mis valores y creencias.

Aunque mi conducta sexual es irresponsable o imprudente, tengo dificultades para detenerla.

Siento como si mi conducta sexual me llevara en una dirección hacia la que no quiero ir.

Hacer cualquier cosa relacionada con el sexo me ayuda a manejar mi estrés.

El sexo es para mí una forma de manejar el malestar emocional que siento.

Sexualmente, me comporto de formas que creo que están mal.

Uso el sexo como una forma de ayuda para afrontar mis problemas.

\begin{tabular}{|c|c|c|}
\hline $2.10(1.14)$ & $1.59(0.80)$ & $-1.71(0.092)$ \\
\hline $1.65(0.93)$ & $1.41(0.80)$ & $-0.93(0.355)$ \\
\hline $1.67(1.05)$ & $1.24(0.44)$ & $-2.38(0.021)$ \\
\hline $1.51(0.67)$ & $1.59(0.94)$ & $0.38(0.709)$ \\
\hline $1.98(1.18)$ & $1.94(0.75)$ & $-0.13(0.898)$ \\
\hline $112(0.38)$ & $1.12(0.49)$ & $0.00(0.999)$ \\
\hline $1.18(0.39)$ & $1.24(0.75)$ & $0.42(0.676)$ \\
\hline $1.33(0.65)$ & $1.29(0.59)$ & $-0.22(0.827)$ \\
\hline $1.92(0.96)$ & $1.88(0.86)$ & $-0.15(0.881)$ \\
\hline $1.61(0.90)$ & $1.47(0.87)$ & $-0.55(0.584)$ \\
\hline $1.31(0.71)$ & $1.41(0.87)$ & $0.467(0.642)$ \\
\hline $1.49(0.86)$ & $1.35(0.70)$ & $-0.60(0.553)$ \\
\hline
\end{tabular}

\section{Discusión}

Con todo, el presente estudio ha analizado el patrón de uso de las aplicaciones de citas y motivaciones en el colectivo LGB, en comparación con la población heterosexual, desde una perspectiva de género. Para ello, además, se han tenido en cuenta variables tan relevantes como la autoestima, la regulación emocional y el valor otorgado a la sensualidad y sexualidad en las relaciones sexuales y de pareja.

En primer término, en línea con pasados estudios, se observa cómo la población LGB utiliza en mayor medida las aplicaciones de contactos y lo hace con más frecuencia que la población heterosexual. Tal y como afirman Martínez-Gómez et al. (2020), los nuevos espacios de socialización que proporcionan los entornos online y más concretamente las aplicaciones, podrían representar un contexto facilitador de las relaciones afectivo-sexuales entre 
aquellas personas alejadas de la heteronormatividad. Aspectos ya señalados, como percibir un entorno más seguro, anónimo y confidencial podrían favorecer dicha experiencia (Chan, 2017).

Probablemente este mayor uso de las aplicaciones favorece que, como muestra nuestro estudio aun sin diferencias significativas, las personas LGB tiendan a hablar con más personas poco conocidas sobre su experiencia con las aplicaciones de citas. Por el contrario, en el caso de la población heterosexual esta información se compartiría, exclusivamente, con personas de su entorno cercano. En este sentido, parecería que entre personas del colectivo LGB, el uso de las apps estaría más normalizado, caracterizándose como un espacio libre de discriminación. En cualquier caso, tanto en población heterosexual como LGB habría personas que no compartirían con nadie su experiencia con las apps de citas. Este fenómeno, dificultaría la propia intervención sobre dichas conductas, por lo que cabría realizar un esfuerzo diferencial para generar un clima de confianza y confidencialidad con aquellas personas usuarias que podrían ocultar, en los procesos de intervención, su participación en las aplicaciones de citas. Esto constituiría un aspecto fundamental de abordaje, si se tienen en cuenta tanto los posibles beneficios de un uso saludable como los perjuicios asociados a un uso de riesgo (Bull y McFlare, 2000; Timmermans y De Caluwé, 2017; Weinstein et al., 2015).

En cuanto a los propósitos, si bien ambos grupos de población muestran similitudes en las motivaciones reportadas, entre la población LGB se observaría un mayor protagonismo de la necesidad de hacer amigos y curiosear. Este fenómeno, podría asociarse a hallazgos previos relativos a otros contextos sexuales online en los que la población LGB planteaba entre los beneficios, la posibilidad de contactar con personas que compartieran sus gustos e intereses (Taylor et al., 2017; Timmermans y De Caluwé, 2017). En línea con el planteamiento de Cass (1979), este proceso podría relacionarse con la fase del proceso identitario y exploración, donde las personas LGB tienen la necesidad de experimentación, exploración e intimidad. Esta fase se caracteriza por la búsqueda de apoyos de socialización para experimentar con los iguales. En este sentido, las apps supondrían nuevos lugares de encuentro LGB, produciéndose un espacio más seguro para conocer gente más allá del nivel afectivosexual (Taylor et al., 2017; Timmermans y De Caluwé, 2017). Al mismo tiempo, la relevancia de satisfacer la curiosidad sobre algunos aspectos podría derivarse de la dificultad que, en muchas ocasiones, las personas LGB tienen para encontrar respuestas adaptadas a sus necesidades de información (Gil-Borrelli et al., 2017).

Respecto a las interacciones con otras personas, un porcentaje considerable de la población heterosexual y LGB quedaría con otras personas con las que ha contactado mediante una aplicación y mantendría relaciones sexuales con ellas, siendo algo superior entre el colectivo LGB. En este sentido, dado el incremento de ITS asociadas a este fenómeno (Klein y Tilley, 2012), cabría dedicar más esfuerzo a las estrategias de prevención primaria contextualizadas en estos entornos de interacción sexual y adaptadas a estos colectivos.

Respecto al uso de las aplicaciones de citas dentro del colectivo LGB, los hombres muestran un mayor porcentaje de uso que las mujeres, al igual que ha ocurrido en pasados estu- 
dios (Martínez-Gómez et al., 2020). Entre las personas que las usan, un porcentaje elevado de hombres y mujeres habría quedado con personas a través de estas aplicaciones si bien, a nivel descriptivo, parece que más hombres habrían mantenido una relación sexual con ellas.

En cuanto a las motivaciones para el uso de las apps, los hombres muestran de manera más notable, una mayor intención de satisfacer su excitación sexual. Estos resultados podrían estar relacionados con aquellos estudios que indican cómo una mayor búsqueda de sensaciones sexuales podría favorecer la intención de buscar relaciones sexuales a través de las apps de citas (Chan, 2016). En este contexto, algunos autores (Gil-Llario, Morell-Mengual, Ballester-Arnal, Giménez-García y Castro-Calvo, 2015) mostrarían cómo la búsqueda de sensaciones sexuales estaría más presente entre los hombres del colectivo LGB que entre las mujeres.

$\mathrm{Al}$ mismo tiempo, entre los hombres, se observa una mayor tendencia a informar la motivación de disminuir el estrés. Este resultado iría en consonancia con lo encontrado en algunos estudios (Ballester-Arnal, Gil-Llario, Giménez-García, Castro-Calvo y Cardenas-López, 2017), donde los hombres reportarían un menor autocontrol sobre su propia conducta sexual, en concreto, una mayor persistencia en conductas sexuales que no querían hacer y un mayor fracaso por controlar su conducta sexual. En consecuencia, tal y como señalan estos autores, los hombres presentarían una mayor probabilidad de tener un uso problemático de la sexualidad, en relación con patrones de abuso y regulación emocional.

Aunque no haya significación estadística, también se observa cómo las mujeres muestran mayor puntuación en satisfacer su curiosidad. Puede que dentro de la población LGB, el colectivo de mujeres todavía esté más invisibilizado y necesite acudir a más entornos para poder experimentar un mayor conocimiento. En este sentido, cabría hacer un esfuerzo diferencial en el colectivo de mujeres que, muchas veces, resulta desatendido más allá de las necesidades reproductivas y de planificación familiar.

Además, nuestros hallazgos apoyarían aquellos estudios que muestran una mayor autoestima sexual entre la población LGB usuaria de apps. Cabría analizar con más detalle este resultado para comprobar si dicha autoestima, tal y como proponen algunos autores (Kim, Kwon y Lee, 2009) se erige como una variable facilitadora que dotaría de mayor seguridad a las personas para buscar nuevas citas y, por tanto, incrementaría su probabilidad de ocurrencia o, como señalan otros autores (Ward, 2016), podría ser resultado del propio uso de las apps de citas que ha mostrado mejorar el bienestar emocional de personas LGB, fortaleciendo también su autoestima, al poder experimentar su vivencia afectivo-sexual en un entorno libre de prejuicios.

Al mismo tiempo, los resultados irían en línea con aquellos autores que muestran una mayor motivación por la sexualidad entre las personas que emplean las apps (Chan, 2017). Seguramente, las aplicaciones suponen un nuevo espacio de interacción y descubrimiento en el que poder experimentar sus motivaciones y deseos sexuales, frente a aquellas personas que estarían menos motivadas por este aspecto y no buscarían nuevas fuentes para cubrirlo. Respecto a la regulación emocional, se observa cómo las personas usuarias de apps suelen recurrir con mayor frecuencia a la sexualidad para gestionar las emociones desagradables, 
propósito que también ha sido observado en otros contextos de interacción sexual online (Castro-Calvo, Giménez-García, Gil-Llario y Ballester-Arnal, 2018). Dada la relación que se ha establecido entre la regulación emocional y la adicción al cibersexo, parece fundamental poner un énfasis en la atención de esta variable, tanto a nivel clínico como preventivo.

Todos estos resultados, cabría valorarlos a tenor de algunas limitaciones que presenta nuestro estudio. Por un lado, sería necesario incluir una mayor cantidad de participantes con el fin de poder generalizar los resultados y, si hubiera variabilidad, diferenciar dentro del propio colectivo LGB para visibilizar su propia diversidad según variables como la orientación o la identidad sexual. En este estudio, el número desigual de participantes que se identificó en cada una de las orientaciones no heterosexuales, dificultó que se pudieran hacer comparaciones entre ellas. Además, el uso de los autoinformes podría favorecer la deseabilidad social.

Más allá de estos aspectos, los hallazgos del presente estudio muestran información valiosa para el abordaje de la sexualidad con el colectivo LGB. En concreto, dada la notable participación del colectivo en el entorno de las aplicaciones de citas, parece necesario incorporar este contexto relacional en las estrategias de evaluación e intervención, con el fin de mejorar las competencias del autocuidado físico, psicológico y social de la población. Para ello, parece necesario seguir analizando los patrones de uso, así como la posible existencia de perfiles diferenciales asociados a un uso de riesgo. En línea con otros estudios, en población general (Baltieri, Junqueira-Aguiar, De Oliveira, De Souza-Gatti y De Souza-Aranha, 2015; Castro-Calvo, García-Barba, Gil-Juliá, Morell-Mengual y Ballester-Arnal, 2018), las personas LGB usuarias de apps han mostrado un mayor uso del sexo para aliviar su malestar, si bien todos los grupos de población han reportado emplear las aplicaciones de citas para disminuir su estrés y entretenerse, quizá para aliviar el aburrimiento. Así pues, cabría analizar más profundamente qué papel podría jugar el malestar emocional en el uso de las apps e incluir un componente de intervención sobre estrategias de regulación emocional, tanto a nivel preventivo como en la intervención clínica. Al mismo tiempo, considerando los posibles beneficios encontrados en el uso de las aplicaciones, sería necesario diseñar intervenciones que también fomentaran estos últimos, a través de un uso responsable.

Para todo lo anterior, dados los resultados encontrados, cabría tener en cuenta una perspectiva de género que permita matizar aquellos aspectos diferenciales en el uso de las aplicaciones de citas, posibilitando una mayor eficacia de las intervenciones.

\section{Referencias}

Ansari, A. (2015). Modern Romance. London: Allen Lane.

Ashford, L., Clifton, D. y Kaneda, T. (2006). La juventud mundial. Washington, DC: Population Reference Bureau.

Ballester-Arnal, R., Castro-Calvo, J., Gil-Juliá, B., Giménez-García, C. y Gil-Llario, M.D. (2019). A Validation Study of the Spanish Version of the Hypersexual Behavior Inventory (HBI): Paper-and-Pencil Versus Online Administration. Journal of Sex \& Marital Therapy, 45(4), 283-302. 
Ballester-Arnal, R., Gil-Llario, M. D., Giménez-García, C., Castro-Calvo, J. y Cárdenas-López, G. (2017). Sexuality in the Internet Era: Expressions of Hispanic Adolescent and Young People. Sexual Addiction and Compulsivity, 24(3), 140-155.

Baltieri, D. A., Junqueira-Aguiar, A. S., De Oliveira, V. H., De Souza-Gatti, A. L. y De Souza-Aranha, R. A. (2015). Validation of the Pornography Consumption Inventory in a Sample of Male Brazilian University Students. Journal of Sex and Marital Therapy, 47(6), 649-660.

Bull, S. S. y McFarlane, M. (2000). Soliciting sex on the Internet: What are the risks for sexually transmitted diseases and HIV? Sexually Transmitted Diseases, 27, 545-550.

Cass, V. C. (1979). Homosexuality identity formation: A theoretical model. Journal of Homosexuality, 4(3), $219-235$.

Castro-Calvo, J., García-Barba, M., Gil-Juliá, B., Morell-Mengual, V. y Ballester-Arnal, R. (2018). Motivos para el consumo de cibersexo y su relación con el grado de severidad. International Journal of Developmental and Educational Psychology, 7(1), 93-102.

Castro-Calvo, J., Giménez-García, C., Gil-Llario, M.D. y Ballester-Arnal, R. (2018). Motives to Engage in Online Sexual Activities and Their Links to Excessive and Problematic Use: a Systematic Review. Current Addiction Reports, 5, 491-510.

Chan, L. S. (2017). Who uses dating apps? Exploring the relationships among trust, sensation-seeking, smartphone use, and the intent to use dating apps based on the Integrative Model. Computers in Human Behavior, 72, 246-258.

Daneback, K., Sevcikova, A., Månsson, S. A. y Ross, M. W. (2013). Outcomes of using the internet for sexual purposes: fulfilment of sexual desires. Sexual Health, 10(1), 26-31.

Gil-Borrelli, C., Velasco, C., Iniesta, C., de Beltrán, P., Curto, J. y Latasa, P. (2017). Hacia una salud pública con orgullo: equidad en la salud en las personas lesbianas, gais, bisexuales y trans en España. Gaceta Sanitaria, 31(3), 175-177.

Gil-Llario, M. D., Morell-Mengual, V., Ballester-Arnal, R., Giménez-García, C. y Castro-Calvo, J. (2015). Sexual sensation seeking in Spanish young men and women with different sexual orientations. Journal of Sex and Marital Therapy, 47(5), 525-530.

Grohmann, R. (2016). Não sou/não curto: sentidos midiatizados de masculinidade, feminilidade e classe social nos discursos de apresentação do aplicativo Grindr. Sessões do Imaginário, 21(35), 1-11.

Hamilton, E. G., Franklin, B., Condesa, C. y Cuauhtémoc, A. (2018). Tinder: La Modernidad del Amor. Recuperado de https://www.researchgate.net/publication/340389407_Eduardo_Guadarrama_Hamilton_-Tinder_en_ la_Modernidad_del_Amor_Tinder_La_Modernidad_del_Amor

Ibarra, A. y Pérez, J. (2013). Jóvenes y Facebook: socialidad mediada e interacciones sociales. Socialidades y afectos. Vida cotidiana, nuevas tecnologías y producciones mediáticas. Guadalajara, México: Universidad de Guadalajara.

Johnson, K., Vilceanu, O. y Pontes, M. (2017). Use of Online Dating Websites and Dating Apps: Findings and Implications for LGB Populations. Journal of Marketing Development and Competitiveness, 17(3), 60-66.

Kim, M., Kwon, K. y Lee, M. (2009). Psychological characteristics of internet dating service users: The effect of self-esteem, involvement, and sociability on the use of internet dating services. CyberPsychology \& Behavior, 12(4), 445-449. doi:10.1089/cpb.2008.029

Klein, H. y Tilley, D. L. (2012). Perceptions of HIV risk among Internet-using, HIV-negative barebacking men. American Journal of Men's Health, 6, 280-293.

Martínez-Gómez, N., Cervigón-Carrasco, V., Nicola Piris, Y. y Giménez-García, C. (2020). Diferencias de sexo en población LGTBI: nuevos escenarios de la sexualidad. Ágora de salut (6), 175-180.

Martínez-Rodrigo, E., Segura-García, R. y Sánchez-Martín, L. (2011). El complejo mundo de la interactividad: Emociones y redes sociales. Revista Mediterránea de Comunicación, 2, 171-190.

Latamclick (2018). Estadísticas de Tinder 2018: análisis y datos de uso de la App de citas. Recuperado de https:// www.latamclick.com/estadisticas-de-tinder-2018-analisis-datos/ 
Reid, R. C., Garos, S. y Carpenter, B. N. (2011). Reliability, validity, and psychometric development of the Hypersexual Behavior Inventory in an outpatient sample of men. Sexual Addiction \& Compulsivity, 18, 30-51.

Rothenburg, W. C. y Stroppa, T. (2015). Freedom of expression and hate speech: the discursive conflict in social networks. Revista electrónica do curso de direito, 10(2), 450-468.

Sabella, R. A., Patchin, J. W. y Hinduja, S. (2013). Cyberbullying myths and realities. Computers in Human Behavior, 29(6), 2703-2711.

Smith, A. (2016, 11 de Febrero). 15\% of American Adults Have Used Online Dating Sites or Mobile Dating Apps. Pew Research Center. Recuperado de https://www.pewresearch.org/internet/2016/02/11/15-percent-ofamerican-adults-have-used-online-dating-sites-or-mobile-dating-apps/

Sevi, B., Aral, T. y Eskenazi, T. (2018). Exploring the hook-up app: Low sexual disgust and high sociosexuality predict motivation to use Tinder for casual sex. Personality and Individual Differences, 133, 17-20.

Snell, W. E., Fisher, T. D. y Walters, A. S. (1993). The multidimensional sexuality questionnaire: An objective self-report measure of psychological tendencies associated with human sexuality. Annals of Sex Research, 6(1), 27-55.

Sumter, S. R., Vandenbosch, L. y Ligtenberg, L. (2017). Love me Tinder: Untangling emerging adults' motivations for using the dating application Tinder. Telematics and Informatics, 34(1), 67-78.

Taylor, S., Hutson, J. y Alicea, T. (2017). Social Consequences of Grindr Use: Extending the Internet Enhanced SelfDisclosure Hypothesis. Proceedings Of The $2017 \mathrm{CHI}$ Conference On Human Factors In Computing Systems, 6645-6657. doi: 10.1145/3025453.3025775

Timmermans, E. y De Caluwé, E. (2017). Development and validation of the tinder motives scale (TMS). Computers in Human Behavior, 70, 341-350.

Tomaszewska, P. y Schuster, I. (2020). Comparing Sexuality-Related Cognitions, Sexual Behavior, and Acceptance of Sexual Coercion in Dating App Users and Non-Users. Sexuality Research and Social Policy, 17(2), 188-198.

Vogels, E. (2020, 24 de Marzo). About half of never-married Americans have used an online dating site or app. Pew Research Center. Recuperado de https://www.pewresearch.org/fact-tank/2020/03/24/the-never-beenmarried-are-biggest-users-of-online-dating/

Ward, J. (2016). Swiping, Matching, Chatting: Self-Presentation and Self-Disclosure on Mobile Dating Apps. HUMAN IT, 13(2), 81-95.

Weinstein, A., Zolek, R., Babkin, A., Cohen, K. y Lejoyeux, M. (2015). Factors Predicting Cybersex Use and Difficulties in Forming Intimate Relationships among Male and Female Users of Cybersex. Frontiers In Psychiatry, 6, 1-8. doi: 10.3389/fpsyt.2015.00054 